\title{
A note on equivalence of some Rotfel'd type theorems
}

\author{
Yaxin Gao, Chaojun Yang, Fangyan Lu* \\ Department of Mathematics, Soochow University, Suzhou 215006 China \\ *Corresponding author, e-mail: fylu@suda.edu.cn
}

Received 1 Apr 2019

Accepted 30 Oct 2019

\begin{abstract}
In this note, we prove that some of recent Rotfel'd type inequalities are equivalent, which is an extension of Huang, Wang and Zhang [Linear Multilinear Algebra 66 (2018) 1626-1632]. Among other results, it is shown that if $f:[0, \infty) \rightarrow[0, \infty)$ is a concave function and $A \in \mathbb{M}_{2}\left(\mathbb{M}_{n}\right)$ is a normal matrix with its numerical range contained in a sector: $S_{\alpha}=\{z \in \mathbb{C}: \operatorname{Re} z \geqslant 0,|\operatorname{Im} z| \leqslant(\operatorname{Re} z) \tan \alpha\}$ for some $\alpha \in\left[0, \frac{\pi}{2}\right)$, then $\|f(|A|)\| \leqslant 2\left\|f\left(\frac{\sec \alpha}{2}\left|A_{11}+A_{22}\right|\right)\right\|$ for any unitarily invariant norm $\|\cdot\|$. This inequality improves a recent result of Zhao and Ni [Linear Multilinear Algebra 66 (2018) 410-417].
\end{abstract}

KEYWORDS: Rotfel'd theorem, concave function, unitarily invariant norm, numerical range

MSC2010: 47A63 47A30

\section{INTRODUCTION}

Throughout this paper, let $\mathbb{M}_{n}$ be the set of all $n \times n$ complex matrices. For $A \in \mathbb{M}_{n}$, its singular values are always arranged in decreasing order: $\sigma_{1}(A) \geqslant \sigma_{2}(A) \geqslant \cdots \geqslant \sigma_{n}(A)$. We denote by $\|A\|$ the unitarily invariant norm of $A$, and $|A|=\left(A^{*} A\right)^{1 / 2}$. If $A$ is Hermitian, we enumerate eigenvalues of $A$ in non-increasing order: $\lambda_{1}(A) \geqslant \lambda_{2}(A) \geqslant \cdots \geqslant \lambda_{n}(A)$. Note that tr is the usual trace functional. For two Hermitian matrices $A, B \in \mathbb{M}_{n}$, we use $A \geqslant B(A \leqslant B)$ to mean that $A-B$ is a positive (negative) semidefinite matrix. A matrix $A \in \mathbb{M}_{n}$ is called accretivedissipative if in its Cartesian (or Toeptliz) decomposition, $A=\operatorname{Re} A+i \operatorname{Im} A$, the matrices $\operatorname{Re} A$ and $\operatorname{Im} A$ are positive semidefinite, where $\operatorname{Re} A=\frac{1}{2}\left(A+A^{*}\right)$, $\operatorname{Im} A=\frac{1}{2 i}\left(A-A^{*}\right)$. From Ref. 18 we know, for the Cartesian decomposition of $A$, that $A$ is normal if and only if $\operatorname{Re} A \operatorname{Im} A=\operatorname{Im} A \operatorname{Re} A$.

The numerical range of $A \in \mathbb{M}_{n}$ is defined by

$$
W(A)=\left\{x^{*} A x: x \in \mathbb{C}^{n}, x^{*} x=1\right\} .
$$

For $\alpha \in\left[0, \frac{\pi}{2}\right), S_{\alpha}$ and $S_{\alpha}^{\prime}$ denote, respectively, the sector regions in the complex plane as follows.

$$
S_{\alpha}=\{z \in \mathbb{C}: \operatorname{Re} z \geqslant 0,|\operatorname{Im} z| \leqslant(\operatorname{Re} z) \tan \alpha\}
$$

and

$$
S_{\alpha}^{\prime}=\{z \in \mathbb{C}: \operatorname{Re} z \geqslant 0,0 \leqslant \operatorname{Im} z \leqslant(\operatorname{Re} z) \tan \alpha\} .
$$

Recent studies on matrices with numerical ranges in a sector can be found in Refs. 5, 6, 11-13, 15, 19 and references therein.
Consider a partitioned matrix $A \in \mathbb{M}_{n}$ in the form

$$
A=\left[\begin{array}{ll}
A_{11} & A_{12} \\
A_{21} & A_{22}
\end{array}\right],
$$

where $A_{11}$ and $A_{22}$ are square matrices. By $\mathbb{M}_{2}\left(\mathbb{M}_{n}\right)$ we mean

$\mathbb{M}_{2}\left(\mathbb{M}_{n}\right)=\left\{\left[\begin{array}{ll}A_{11} & A_{12} \\ A_{21} & A_{22}\end{array}\right]: A_{i j} \in \mathbb{M}_{n}, i, j=1,2\right\}$.

Similarly, we can define $\mathbb{M}_{n}\left(\mathbb{M}_{k}\right)$.

In the late 1960s, Rotfel'd proved a famous trace inequality: let $A, B \geqslant 0$ and let $f$ be a non-negative concave function on $[0, \infty)$. Then

$$
\operatorname{tr} f(A+B) \leqslant \operatorname{tr} f(A)+\operatorname{tr} f(B) .
$$

Lee extended the Rotfel'd theorem to a partitioned positive semidefinite matrix ${ }^{10}$.

Theorem 1 Let $A \in \mathbb{M}_{n}$ be a positive semidefinite matrix partitioned as in (1) and let $f:[0, \infty) \rightarrow$ $[0, \infty)$ be a concave function. Then

$$
\|f(A)\| \leqslant\left\|f\left(A_{11}\right)\right\|+\left\|f\left(A_{22}\right)\right\| .
$$

As a further extension of the classic Rotfel'd theorem, Zhang ${ }^{16}$ extended Theorem 1 to matrices with $W(A) \subseteq S_{\alpha}$ for $\alpha \in\left[0, \frac{\pi}{2}\right)$ as follows.

Theorem 2 Let $f:[0, \infty) \rightarrow[0, \infty)$ be a concave function and let $A \in \mathbb{M}_{n}$ with $W(A) \subseteq S_{\alpha}$ for $\alpha \in\left[0, \frac{\pi}{2}\right)$ be partitioned as in (1). Then

$$
\begin{aligned}
\|f(|A|)\| & \leqslant\left\|f\left(\left|A_{11}\right|\right)\right\|+\left\|f\left(\left|A_{22}\right|\right)\right\| \\
& +2\left(\left\|f\left(\tan (\alpha)\left|A_{11}\right|\right)\right\|+\left\|f\left(\tan (\alpha)\left|A_{22}\right|\right)\right\|\right) .
\end{aligned}
$$


Later, Fu and $\mathrm{Liu}^{6}$ obtained another generalization of Theorem 1 as follows.

Theorem 3 Let $f:[0, \infty) \rightarrow[0, \infty)$ be a concave function and let $A \in \mathbb{M}_{n}$ with $W(A) \subseteq S_{\alpha}$ for $\alpha \in\left[0, \frac{\pi}{2}\right)$ be partitioned as in (1). Then

$\|f(|A|)\| \leqslant\left\|f\left(\sec ^{2}(\alpha)\left|A_{11}\right|\right)\right\|+\left\|f\left(\sec ^{2}(\alpha)\left|A_{22}\right|\right)\right\|$.

Hou and Zhang ${ }^{7}$ considered the case: $W(A) \subseteq S_{\alpha}^{\prime}$ for $\alpha \in\left[0, \frac{\pi}{2}\right)$. They derived the following result.

Theorem 4 Let $f:[0, \infty) \rightarrow[0, \infty)$ be a concave function and let $A \in \mathbb{M}_{n}$ with $W(A) \subseteq S_{\alpha}^{\prime}$ for $\alpha \in\left[0, \frac{\pi}{2}\right)$ be partitioned as in (1). Then

$$
\begin{aligned}
\|f(|A|)\| \leqslant & \left\|f\left(\left|A_{11}\right|\right)\right\|+\left\|f\left(\left|A_{22}\right|\right)\right\| \\
& +\left\|f\left(\tan (\alpha)\left|A_{11}\right|\right)\right\|+\left\|f\left(\tan (\alpha)\left|A_{22}\right|\right)\right\| .
\end{aligned}
$$

Let $A$ be normal and $W(A) \subseteq S_{\alpha}, \alpha \in\left[0, \frac{\pi}{2}\right)$. Zhao and $\mathrm{Ni}^{17}$ derived the following result.

Theorem 5 Let $f:[0, \infty) \rightarrow[0, \infty)$ be a concave function and $A \in \mathbb{M}_{n}$ be normal with $W(A) \subseteq S_{\alpha}$ for $\alpha \in\left[0, \frac{\pi}{2}\right)$, and let $A$ be partitioned as in (1). Then

$$
\begin{aligned}
\|f(|A|)\| \leqslant & \left\|f\left(\left|A_{11}\right|\right)\right\|+\left\|f\left(\left|A_{22}\right|\right)\right\| \\
& +\left\|f\left(\tan (\alpha)\left|A_{11}\right|\right)\right\|+\left\|f\left(\tan (\alpha)\left|A_{22}\right|\right)\right\| .
\end{aligned}
$$

Huang et $\mathrm{al}^{8}$ derived the following inequality.

Theorem 6 Let $A \in \mathbb{M}_{n}$ be partitioned as in (1) and let $f:[0, \infty) \rightarrow[0, \infty)$ be a concave function. If $A+$ $A^{*} \geqslant 0$, then

$$
\left\|f\left(\frac{A+A^{*}}{2}\right)\right\| \leqslant\left\|f\left(\left|A_{11}\right|\right)\right\|+\left\|f\left(\left|A_{22}\right|\right)\right\| .
$$

Recently, Yang et al ${ }^{15}$ presented a new refinement of Rotfel'd type inequality as follows.

Theorem 7 Let $f:[0, \infty) \rightarrow[0, \infty)$ be a concave function and $A \in \mathbb{M}_{n}$ be normal with $W(A) \subseteq S_{\alpha}$ for $\alpha \in\left[0, \frac{\pi}{2}\right)$ and let $A$ be partitioned as in (1). Then

$$
\|f(|A|)\| \leqslant\left\|f\left(\sec (\alpha)\left|A_{11}\right|\right)\right\|+\left\|f\left(\sec (\alpha)\left|A_{22}\right|\right)\right\| .
$$

Zhao and Ni presented an extension of Rotfel'd theorem as follows ${ }^{17}$.

Theorem 8 Let $f:[0, \infty) \rightarrow[0, \infty)$ be a concave function, and $A \in \mathbb{M}_{2}\left(\mathbb{M}_{n}\right)$ be a positive semidefinite matrix, and let $A$ be partitioned as in (2). Then

$$
\|f(2 A)\| \leqslant 2\left\|f\left(A_{11}\right)+f\left(A_{22}\right)\right\| .
$$

In this note, we show that Theorems 1-7 are equivalent, which is an an extension of Huang et $\mathrm{al}^{8}$. In addition, we present a new inequality that can be viewed as a generalization of Theorem 8 .

\section{MAIN RESULTS}

We observe that. If $f:[0, \infty) \rightarrow[0, \infty)$ is concave, then

$$
0 \leqslant A \leqslant B \Longrightarrow\|f(A)\| \leqslant\|f(B)\| .
$$

Before we give the main results, let us present the following lemmas that will be useful later.

Lemma 1 (Ref. 3) Let $A \in \mathbb{M}_{n}$. Then

$$
\lambda_{j}(\operatorname{Re} A) \leqslant \sigma_{j}(A), \quad j=1,2, \ldots, n .
$$

The above inequality implies that there exists a unitary matrix $U \in \mathbb{M}_{n}$ such that

$$
\operatorname{Re} A \leqslant U|A| U^{*} .
$$

Zhao and $\mathrm{Ni}^{17}$ presented a decomposition lemma as follows.

Lemma 2 Let $A \in \mathbb{M}_{2}\left(\mathbb{M}_{n}\right)$ be a positive semidefinite matrix, and let $A$ be partitioned as in (2). Then there exist unitary matrices $U, V \in \mathbb{M}_{2}\left(\mathbb{M}_{n}\right)$ such that

$A=\frac{1}{2}\left\{U\left[\begin{array}{cc}A_{11}+A_{22} & 0 \\ 0 & 0\end{array}\right] U^{*}+V\left[\begin{array}{cc}0 & 0 \\ 0 & A_{11}+A_{22}\end{array}\right] V^{*}\right\}$.

The next lemma was obtained by Aujla and Bourin ${ }^{2}$.

Lemma 3 Let $f:[0, \infty) \rightarrow[0, \infty)$ be a concave function, and $A, B \in \mathbb{M}_{n}$ be positive semidefinite matrices. Then there exist unitary matrices $U, V \in \mathbb{M}_{n}$ such that

$$
f(A+B) \leqslant U f(A) U^{*}+V f(B) V^{*} .
$$

Bourin and Lee ${ }^{4}$ obtained the following important inequality.

Lemma 4 Let $A, B \geqslant 0$ and $f:[0, \infty) \rightarrow[0, \infty)$ be a concave function. Then

$$
\|f(A+B)\| \leqslant\|f(A)+f(B)\| .
$$

The following lemma was obtained by Yang et $\mathrm{al}^{15}$.

Lemma 5 Let $A=R+i S$ be the Cartesian decomposition of $A$ with $W(A) \subseteq S_{\alpha}$ for $\alpha \in\left[0, \frac{\pi}{2}\right)$. If $R S=S R$ (i.e., $A$ is normal), then

$$
|A| \leqslant \sec (\alpha) R .
$$

Now we are ready to give the first main result. 
Theorem 9 Let $f:[0, \infty) \rightarrow[0, \infty)$ be a concave function and $A \in \mathbb{M}_{2}\left(\mathbb{M}_{n}\right)$ with $W(A) \subseteq S_{\alpha}$ for $\alpha \in$ $\left[0, \frac{\pi}{2}\right)$, and let $A$ be partitioned as in (2). If $A=R+i S$ is the Cartesian decomposition of $A$ with $R S=S R$, then

$$
\|f(|A|)\| \leqslant 2\left\|f\left(\frac{\sec \alpha}{2}\left|A_{11}+A_{22}\right|\right)\right\| .
$$

Proof: We suppose $f(0)=0$, the general case follows directly by using Lee's approach ${ }^{10}$. Consider the Cartesian decomposition $A=R+i S$, where

$$
R=\left[\begin{array}{ll}
R_{11} & R_{12} \\
R_{21} & R_{22}
\end{array}\right] \text { and } S=\left[\begin{array}{ll}
S_{11} & S_{12} \\
S_{21} & S_{22}
\end{array}\right] .
$$

As $R S=S R$, It follows from Lemma 5 that $|A| \leqslant \sec (\alpha) R$. This gives

$$
\begin{aligned}
|A| \leqslant & \frac{\sec \alpha}{2}\left\{U_{1}\left[\begin{array}{cc}
R_{11}+R_{22} & 0 \\
0 & 0
\end{array}\right] U_{1}^{*}+V_{1}\left[\begin{array}{cc}
0 & 0 \\
0 & R_{11}+R_{22}
\end{array}\right] V_{1}^{*}\right\} \\
\leqslant & \frac{\sec \alpha}{2}\left\{U_{1} U_{2}\left[\begin{array}{cc}
\left|R_{11}+R_{22}+i\left(S_{11}+S_{22}\right)\right| & 0 \\
0 & 0
\end{array}\right] U_{2}^{*} U_{1}^{*}\right. \\
& \left.+V_{1} V_{2}\left[\begin{array}{cc}
0 & \left|R_{11}+R_{22}+i\left(S_{11}+S_{22}\right)\right|
\end{array}\right] V_{2}^{*} V_{1}^{*}\right\} \\
= & \frac{\sec \alpha}{2}\left\{U_{1} U_{2}\left[\begin{array}{cc}
\left|A_{11}+A_{22}\right| & 0 \\
0 & 0
\end{array}\right] U_{2}^{*} U_{1}^{*}\right. \\
& \left.+V_{1} V_{2}\left[\begin{array}{ccc}
0 & 0 \\
0 & \left|A_{11}+A_{22}\right|
\end{array}\right] V_{2}^{*} V_{1}^{*}\right\} \\
= & U_{1} U_{2}\left[\begin{array}{cc}
\frac{\sec \alpha}{2}\left|A_{11}+A_{22}\right| & 0 \\
0 & 0
\end{array}\right] U_{2}^{*} U_{1}^{*} \\
& +V_{1} V_{2}\left[\begin{array}{cc}
0 & 0 \\
0 & \frac{\sec \alpha}{2}\left|A_{11}+A_{22}\right|
\end{array}\right] V_{2}^{*} V_{1}^{*},
\end{aligned}
$$

where the first and the second equalities are obtained by Lemma 2 and Lemma 1, with corresponding unitary matrices $U_{1}, V_{1}$, and $U_{2}, V_{2}$, respectively.

By (3), Lemma 3 and the triangle inequality,

$$
\begin{aligned}
\|f(|A|)\| \leqslant & \| U_{3} U_{1} U_{2}\left[\begin{array}{cc}
f\left(\frac{\sec \alpha}{2}\left|A_{11}+A_{22}\right|\right) & 0 \\
0 & 0
\end{array}\right] U_{2}^{*} U_{1}^{*} U_{3}^{*} \\
& +V_{3} V_{1} V_{2}\left[\begin{array}{cc}
0 & 0 \\
0 & f\left(\frac{\sec \alpha}{2}\left|A_{11}+A_{22}\right|\right)
\end{array}\right] V_{2}^{*} V_{1}^{*} V_{3}^{*} \| \\
\leqslant & \left\|\left[\begin{array}{cc}
f\left(\frac{\sec \alpha}{2}\left|A_{11}+A_{22}\right|\right) & 0 \\
0 & 0
\end{array}\right]\right\| \\
& +\left\|\left[\begin{array}{cc}
0 & 0 \\
0 & f\left(\frac{\sec \alpha}{2}\left|A_{11}+A_{22}\right|\right)
\end{array}\right]\right\| \\
= & 2\left\|f\left(\frac{\sec \alpha}{2}\left|A_{11}+A_{22}\right|\right)\right\|,
\end{aligned}
$$

where $U_{3}, V_{3}$ are unitary matrices in Lemma 3.

Remark 1 In Theorem 9, we can present another form of (4) as

$$
\|f(2|A|)\| \leqslant 2\left\|f\left(\sec (\alpha)\left|A_{11}+A_{22}\right|\right)\right\| .
$$

For a positive semidefinite matrix $A$, (5) and Lemma 4 give

$$
\|f(2 A)\| \leqslant 2\left\|f\left(A_{11}+A_{22}\right)\right\| \leqslant 2\left\|f\left(A_{11}\right)+f\left(A_{22}\right)\right\| .
$$

Thus Theorem 9 can be considered as a natural generalization of Theorem 8 .

Remark 2 Putting $f(t)=t$ in Theorem 9, we obtain the inequalities

$$
\begin{aligned}
\|f(|A|)\| & =\||A|\| \\
& \leqslant 2\left\|f\left(\frac{\sec \alpha}{2}\left|A_{11}+A_{22}\right|\right)\right\| \\
& =\sec (\alpha)\left\|\left|A_{11}+A_{22}\right|\right\|=\sec (\alpha)\left\|A_{11}+A_{22}\right\| \\
& \leqslant \sec (\alpha)\left(\left\|A_{11}\right\|+\left\|A_{22}\right\|\right) \\
& =\sec (\alpha)\left(\left\|A_{11}|\|+\|| A_{22} \mid\right\|\right) \\
& =\left\|f\left(\sec (\alpha)\left|A_{11}\right|\right)\right\|+\left\|f\left(\sec (\alpha)\left|A_{22}\right|\right)\right\| .
\end{aligned}
$$

Under this condition, Theorem 9 is a refinement of Theorem 7.

We borrow an example from Ref. 15 to show that the equality in (4) may happen.

Example 1 Let $f(t)=t$ be concave and

$$
A=\left[\begin{array}{cc}
\cos \alpha & \sin \alpha \\
-\sin \alpha & \cos \alpha
\end{array}\right], \quad \alpha \in\left[0, \frac{\pi}{2}\right) .
$$

By simple calculation, we have

$$
\sigma_{1}(A)=\sigma_{2}(A)=1 .
$$

Specifying the unitarily invariant norm in this example to the trace norm $\|\cdot\|_{\text {tr }}$. Thus we have $\||A|\|_{\text {tr }}=$ $\sigma_{1}(A)+\sigma_{2}(A)=2$ and $\left\|\left|A_{11}+A_{22}\right|\right\|_{\mathrm{tr}}=2 \cos \alpha$, which leads to

$$
\|f(|A|)\|_{\text {tr }}=\||A|\|_{\text {tr }}=2\left\|\frac{\sec \alpha}{2}\left|A_{11}+A_{22}\right|\right\|_{\text {tr }}=2 .
$$

Corollary 1 Let $f:[0, \infty) \rightarrow[0, \infty)$ be a concave function, and $A \in \mathbb{M}_{2}\left(\mathbb{M}_{n}\right)$ with $W(A) \subseteq S_{\alpha}$ for $\alpha \in$ $\left[0, \frac{\pi}{2}\right)$, and let $A$ be partitioned as in (2). If $A=R+$ iS is the Cartesian decomposition of $A$ with $R S=S R$, then

$$
\|f(|A|)\| \leqslant 2\left(\left\|f\left(\frac{\sec \alpha}{2}\left|A_{11}\right|\right)\right\|+\left\|f\left(\frac{\sec \alpha}{2}\left|A_{22}\right|\right)\right\|\right) .
$$

Proof: It follows from Theorem 9 that there exists unitary matrices $U, V \in \mathbb{M}_{2}\left(\mathbb{M}_{n}\right)$ such that

$$
\begin{aligned}
\|f(|A|)\| & \leqslant 2\left\|f\left(\frac{\sec \alpha}{2}\left|A_{11}+A_{22}\right|\right)\right\| \\
& \leqslant 2\left\|f\left(\frac{\sec \alpha}{2}\left(U\left|A_{11}\right| U^{*}+V\left|A_{22}\right| V^{*}\right)\right)\right\| \\
& \leqslant 2\left\|U f\left(\frac{\sec \alpha}{2}\left|A_{11}\right|\right) U^{*}+V f\left(\frac{\sec \alpha}{2}\left|A_{22}\right|\right) V^{*}\right\| \\
& \leqslant 2\left(\left\|f\left(\frac{\sec \alpha}{2}\left|A_{11}\right|\right)\right\|+\left\|f\left(\frac{\sec \alpha}{2}\left|A_{22}\right|\right)\right\|\right),
\end{aligned}
$$


where the third inequality is from Lemma 4.

We can also obtain Corollary 1 by utilizing Theorem 7 as follows.

$$
\begin{aligned}
\|f(|A|)\| & \leqslant\left\|f\left(\sec (\alpha)\left|A_{11}\right|\right)\right\|+\left\|f\left(\sec (\alpha)\left|A_{22}\right|\right)\right\| \\
& \leqslant 2\left(\left\|f\left(\frac{\sec \alpha}{2}\left|A_{11}\right|\right)\right\|+\left\|f\left(\frac{\sec \alpha}{2}\left|A_{22}\right|\right)\right\|\right) .
\end{aligned}
$$

Note that matrix $A$ is accretive-dissipative if and only if $W\left(\mathrm{e}^{-\pi / 4} A\right) \subset S_{\pi / 4}$. Let $\alpha=\pi / 4$ be in Corollary 1 , we obtain

$$
\|f(|A|)\| \leqslant 2\left(\left\|f\left(\frac{\sqrt{2}}{2}\left|A_{11}\right|\right)\right\|+\left\|f\left(\frac{\sqrt{2}}{2}\left|A_{22}\right|\right)\right\|\right),
$$

which coincides with (3.1) in Ref. 16. If we put $\alpha=$ $\pi / 4$ in Theorem 9 , then

$$
\|f(|A|)\| \leqslant 2\left\|f\left(\frac{\sqrt{2}}{2}\left|A_{11}+A_{22}\right|\right)\right\| .
$$

We give a refinement of Corollary 1 without the normality assumption on $A$ in the following theorem.

Theorem 10 Let $f:[0, \infty) \rightarrow[0, \infty)$ be a concave function, and $A \in \mathbb{M}_{n}$ with $W(A) \subseteq S_{\alpha}$ for $\alpha \in\left[0, \frac{\pi}{2}\right)$, and let $A$ be partitioned as in (1). Then

$$
\|f(|A|)\| \leqslant 2\left(\left\|f\left(\frac{\sec \alpha}{2}\left|A_{11}\right|\right)\right\|+\left\|f\left(\frac{\sec \alpha}{2}\left|A_{22}\right|\right)\right\|\right) .
$$

Proof: Let $A=U|A|$ be the polar decomposition of $A$, and $A=R+i S$ be the Cartesian decomposition of $A$ with $R, S$ being partitioned as in (1). Thus by Ref. 4, there exist unitary matrices $U_{1}, V_{1}$ such that

$$
R=\left[U_{1}\left[\begin{array}{cc}
R_{11} & 0 \\
0 & 0
\end{array}\right] U_{1}^{*}+V_{1}\left[\begin{array}{cc}
0 & 0 \\
0 & R_{22}
\end{array}\right] V_{1}^{*}\right]
$$

which gives

$$
\begin{aligned}
& |A| \leqslant \frac{\sec \alpha}{2}\left(R+U^{*} R U\right) \\
& =\frac{\sec \alpha}{2}\left\{U_{1}\left[\begin{array}{cc}
R_{11} & 0 \\
0 & 0
\end{array}\right] U_{1}^{*}+V_{1}\left[\begin{array}{cc}
0 & 0 \\
0 & R_{22}
\end{array}\right] V_{1}^{*}\right. \\
& \left.+U^{*} U_{1}\left[\begin{array}{cc}
R_{11} & 0 \\
0 & 0
\end{array}\right] U_{1}^{*} U+U^{*} V_{1}\left[\begin{array}{cc}
0 & 0 \\
0 & R_{22}
\end{array}\right] V_{1}^{*} U\right\} \\
& \leqslant \frac{\sec \alpha}{2}\left\{U_{1} U_{2}\left[\begin{array}{cc}
\left|R_{11}+i S_{11}\right| & 0 \\
0 & 0
\end{array}\right] U_{2}^{*} U_{1}^{*}\right. \\
& +V_{1} V_{2}\left[\begin{array}{cc}
0 & 0 \\
0 & \left|R_{22}+i S_{22}\right|
\end{array}\right] V_{2}^{*} V_{1}^{*} \\
& +U^{*} U_{1} U_{2}\left[\begin{array}{cc}
\left|R_{11}+i S_{11}\right| & 0 \\
0 & 0
\end{array}\right] U_{2}^{*} U_{1}^{*} U \\
& \left.+U^{*} V_{1} V_{2}\left[\begin{array}{cc}
0 & 0 \\
0 & \left|R_{22}+i S_{22}\right|
\end{array}\right] V_{2}^{*} V_{1}^{*} U\right\} \\
& =\frac{\sec \alpha}{2}\left\{U_{1} U_{2}\left[\begin{array}{cc}
\left|A_{11}\right| & 0 \\
0 & 0
\end{array}\right] U_{2}^{*} U_{1}^{*}+V_{1} V_{2}\left[\begin{array}{cc}
0 & 0 \\
0 & \left|A_{22}\right|
\end{array}\right] V_{2}^{*} V_{1}^{*}\right. \\
& \left.+U^{*} U_{1} U_{2}\left[\begin{array}{cc}
\left|A_{11}\right| & 0 \\
0 & 0
\end{array}\right] U_{2}^{*} U_{1}^{*} U+U^{*} V_{1} V_{2}\left[\begin{array}{cc}
0 & 0 \\
0 & \left|A_{22}\right|
\end{array}\right] V_{2}^{*} V_{1}^{*} U\right\} \\
& =U_{1} U_{2}\left[\begin{array}{cc}
\frac{\sec \alpha}{2}\left|A_{11}\right| & 0 \\
0 & 0
\end{array}\right] U_{2}^{*} U_{1}^{*}+V_{1} V_{2}\left[\begin{array}{cc}
0 & 0 \\
0 & \frac{\sec \alpha}{2}\left|A_{22}\right|
\end{array}\right] V_{2}^{*} V_{1}^{*} \\
& +U^{*} U_{1} U_{2}\left[\begin{array}{cc}
\frac{\sec \alpha}{2}\left|A_{11}\right| & 0 \\
0 & 0
\end{array}\right] U_{2}^{*} U_{1}^{*} U \\
& +U^{*} V_{1} V_{2}\left[\begin{array}{cc}
0 & 0 \\
0 & \frac{\sec \alpha}{2}\left|A_{22}\right|
\end{array}\right] V_{2}^{*} V_{1}^{*} U,
\end{aligned}
$$

where the first inequality is obtained by the previous equality ${ }^{1}$ and the second inequality is obtained by Lemma 1 with unitary matrices $U_{1}, V_{1}$, and $U_{2}, V_{2}$, respectively.

By (3) and Lemma 3, we have

$$
\begin{aligned}
\|f(|A|)\| \leqslant & \| U_{3} U_{1} U_{2}\left[\begin{array}{cc}
f\left(\frac{\sec \alpha}{2}\left|A_{11}\right|\right) & 0 \\
0 & 0
\end{array}\right] U_{2}^{*} U_{1}^{*} U_{3}^{*} \\
& +V_{3} V_{1} V_{2}\left[\begin{array}{cc}
0 & 0 \\
0 & f\left(\frac{\sec \alpha}{2}\left|A_{22}\right|\right)
\end{array}\right] V_{2}^{*} V_{1}^{*} V_{3}^{*} \\
& +U_{3} U^{*} U_{1} U_{2}\left[\begin{array}{cc}
f\left(\frac{\sec \alpha}{2}\left|A_{11}\right|\right) & 0 \\
0 & 0
\end{array}\right] U_{2}^{*} U_{1}^{*} U U_{3}^{*} \\
& +V_{3} U^{*} V_{1} V_{2}\left[\begin{array}{cc}
0 & 0 \\
0 & f\left(\frac{\sec \alpha}{2}\left|A_{22}\right|\right)
\end{array}\right] V_{2}^{*} V_{1}^{*} U V_{3}^{*} \|, \\
\|f(|A|)\| \leqslant & 2\left\|\left[\begin{array}{cc}
f\left(\frac{\sec \alpha}{2}\left|A_{11}\right|\right) & 0 \\
0 & 0
\end{array}\right]\right\| \\
& +2\left\|\left[\begin{array}{cc}
0 & 0 \\
0 & f\left(\frac{\sec \alpha}{2}\left|A_{22}\right|\right)
\end{array}\right]\right\| \\
= & 2\left(\left\|f\left(\frac{\sec \alpha}{2}\left|A_{11}\right|\right)\right\|+\left\|f\left(\frac{\sec \alpha}{2}\left|A_{22}\right|\right)\right\|\right),
\end{aligned}
$$


in which $U_{3}, V_{3}$ correspond to the unitary matrices in Lemma 3.

Letting $f(t)=t$ in Theorem 10, we obtain the following corollary.

Corollary 2 Let $A$ be partitioned as in (2) with $W(A) \subseteq S_{\alpha}$ for $\alpha \in\left[0, \frac{\pi}{2}\right)$. If $A$ is normal, then

$$
\|A\| \leqslant \sec (\alpha)\left\|A_{11}+A_{22}\right\| .
$$

Next we shall extend inequality (6) to a higher number of blocks. First of all, let us introduce some relevant conceptions.

A matrix $A=\left(A_{i j}\right)_{i, j=1}^{n} \in \mathbb{M}_{n}\left(\mathbb{M}_{\mathrm{k}}\right)$ is said to be positive partial transpose (PPT) if $A$ is positive semidefinite, and its partial transpose $A^{\tau}=\left(A_{j i}\right)_{i, j=1}^{n}$ is also positive semidefinite.

In Ref. 9, Kuai defined a new conception called sectorial partial transpose (SPT). A matrix $A=$ $\left(A_{i j}\right)_{i, j=1}^{n} \in \mathbb{M}_{n}\left(\mathbb{M}_{\mathrm{k}}\right)$ is said to be SPT if $W(A) \subseteq S_{\alpha}$ and $W\left(A^{\tau}\right) \subseteq S_{\alpha}$.

Lemma 6 (Ref. 9) If $A$ is SPT, then $\operatorname{Re} A$ is PPT.

Lemma 7 (Ref. 19) Let $A \in \mathbb{M}_{n}$ be such that $W(A) \subseteq$ $S_{\alpha}$. Then

$$
\|A\| \leqslant \sec (\alpha)\|\operatorname{Re} A\| .
$$

Lemma 8 (Ref. 14) Let $A=\left(A_{i j}\right)_{i, j=1}^{n} \in \mathbb{M}_{n}\left(\mathbb{M}_{\mathrm{k}}\right)$ be a PPT matrix. Then

$$
\|A\| \leqslant\left\|\sum_{i=1}^{n} A_{i i}\right\| .
$$

We note that the following theorem is an extension of Corollary 2 and Lemma 8 to sector matrices.

Theorem 11 Let $A=\left(A_{i j}\right)_{i, j=1}^{n} \in \mathbb{M}_{n}\left(\mathbb{M}_{\mathrm{k}}\right)$ be an $S P T$ matrix. Then

$$
\|A\| \leqslant \sec (\alpha)\left\|\sum_{i=1}^{n} A_{i i}\right\| .
$$

Proof: As $A$ is SPT, we obtain by Lemma 6 that $\operatorname{Re} A$ is PPT. Hence we have

$$
\begin{array}{rlr}
\|A\| & \leqslant \sec (\alpha)\|\operatorname{Re} A\| & \text { (by Lemma 7) } \\
& \leqslant \sec (\alpha)\left\|\sum_{i=1}^{n} \operatorname{Re} A_{i i}\right\| \quad \text { (by Lemma 8) } \\
& =\sec (\alpha)\left\|\operatorname{Re}\left(\sum_{i=1}^{n} A_{i i}\right)\right\| \leqslant \sec (\alpha)\left\|\sum_{i=1}^{n} A_{i i}\right\| .
\end{array}
$$

Next we give our second main result, which proves the equivalence of some recent Rotfel'd type theorems.
Theorem 12 Let $f:[0, \infty) \rightarrow[0, \infty)$ be a concave function and $A \in \mathbb{M}_{n}$ be partitioned as in (1). The following statements are equivalent.

(a) If $A \in \mathbb{M}_{n}$ be a positive semidefinite matrix, then ${ }^{10}$

$$
\|f(A)\| \leqslant\left\|f\left(A_{11}\right)\right\|+\left\|f\left(A_{22}\right)\right\| .
$$

(b) If $A+A^{*} \geqslant 0$, then ${ }^{8}$

$$
\left\|f\left(\frac{A+A^{*}}{2}\right)\right\| \leqslant\left\|f\left(\left|A_{11}\right|\right)\right\|+\left\|f\left(\left|A_{22}\right|\right)\right\| .
$$

(c) If $W(A) \subseteq S_{\alpha}^{\prime}$ for $\alpha \in\left[0, \frac{\pi}{2}\right)$, then ${ }^{7}$

$$
\begin{aligned}
\|f(|A|)\| & \leqslant\left\|f\left(\left|A_{11}\right|\right)\right\|+\left\|f\left(\left|A_{22}\right|\right)\right\| \\
& +\left\|f\left(\tan (\alpha)\left|A_{11}\right|\right)\right\|+\left\|f\left(\tan (\alpha)\left|A_{22}\right|\right)\right\| .
\end{aligned}
$$

(d) If $A$ is normal and $W(A) \subseteq S_{\alpha}$ for $\alpha \in\left[0, \frac{\pi}{2}\right.$ ), then ${ }^{17}$

$$
\begin{aligned}
\|f(|A|)\| & \leqslant\left\|f\left(\left|A_{11}\right|\right)\right\|+\left\|f\left(\left|A_{22}\right|\right)\right\| \\
& +\left\|f\left(\tan (\alpha)\left|A_{11}\right|\right)\right\|+\left\|f\left(\tan (\alpha)\left|A_{22}\right|\right)\right\| .
\end{aligned}
$$

(e) If $W(A) \subseteq S_{\alpha}$ for $\alpha \in\left[0, \frac{\pi}{2}\right)$, then ${ }^{19}$

$$
\begin{aligned}
& \|f(|A|)\| \leqslant\left\|f\left(\left|A_{11}\right|\right)\right\|+\left\|f\left(\left|A_{22}\right|\right)\right\| \\
& \quad+2\left(\left\|f\left(\tan (\alpha)\left|A_{11}\right|\right)\right\|+\left\|f\left(\tan (\alpha)\left|A_{22}\right|\right)\right\|\right) .
\end{aligned}
$$

(f) If $A$ is normal and $W(A) \subseteq S_{\alpha}$ for $\alpha \in\left[0, \frac{\pi}{2}\right)$, then ${ }^{15}$

$$
\|f(|A|)\| \leqslant\left\|f\left(\sec (\alpha)\left|A_{11}\right|\right)\right\|+\left\|f\left(\sec (\alpha)\left|A_{22}\right|\right)\right\| .
$$

(g) If $W(A) \subseteq S_{\alpha}$ for $\alpha \in\left[0, \frac{\pi}{2}\right)$, then ${ }^{6}$

$$
\|f(|A|)\| \leqslant\left\|f\left(\sec ^{2}(\alpha)\left|A_{11}\right|\right)\right\|+\left\|f\left(\sec ^{2}(\alpha)\left|A_{22}\right|\right)\right\| .
$$

Proof: The equivalence from (a)-(e) was shown by Huang et $\mathrm{al}^{8}$, we thus only need to prove (b) $\Longrightarrow$ (f), (b) $\Longrightarrow$ ( (), (f) $\Longrightarrow$ (a), and ( $\mathrm{g}) \Longrightarrow$ (a).

(b) $\Longrightarrow$ (f): Consider the Cartesian decomposition $A=R+i S$. It follows from Lemma 5 that

$$
|A| \leqslant \sec (\alpha) R
$$

Then, by (3) and (b), we have

$$
\begin{aligned}
\|f(|A|)\| & \leqslant\|f(\sec (\alpha) R)\| \\
& =\left\|f\left(\frac{(\sec (\alpha) A)+(\sec (\alpha) A)^{*}}{2}\right)\right\| \\
& \leqslant\left\|f\left(\sec (\alpha)\left|A_{11}\right|\right)\right\|+\left\|f\left(\sec (\alpha)\left|A_{22}\right|\right)\right\| .
\end{aligned}
$$


(b) $\Longrightarrow(\mathrm{g})$ : For the Cartesian decomposition $A=R+i S$, it follows from Ref. 5 that there exists a unitary matrix $U \in \mathbb{M}_{n}$ such that

$$
|A| \leqslant \sec ^{2}(\alpha) U R U^{*},
$$

and the rest of the proof is the same as above.

(f) $\Longrightarrow$ (a): For a positive semidefinite matrix $A$, we have $\alpha=0$ in (f), which implies (a) directly. Similarly, we obtain $(\mathrm{g}) \Longrightarrow$ (a).

Apparently, Theorem 12 is an extension of Huang et $\mathrm{al}^{8}$ (Theorem 3.1).

Acknowledgements: This study is supported by the National Natural Science Foundation of China (No. 11571247).

\section{REFERENCES}

1. Alakhrass M (2019) A note on sectorial matrices. Linear Multilinear Algebra.

2. Aujla J, Bourin JC (2007) Eigenvalues inequalities for convex and log-convex function. Linear Algebra Appl 424, 25-35.

3. Bhatia R (1997) Matrix Analysis, Springer.

4. Bourin JC, Lee EY (2012) Unitary orbits of Hermitian operators with convex or concave functions. Bull London Math Soc 44, 1085-1102.

5. Drury S, Lin M (2014) Singular value inequalities for matrices with numerical ranges in a sector. Oper Matrices 8, 1143-1148.

6. Fu X, Liu Y (2016) Rotfel'd inequality for partitioned matrices with numerical ranges in a sector. Linear Multilinear Algebra 64, 105-109.
7. Hou L, Zhang D (2017) Concave functions of partitioned matrices with numerical ranges in a sector. Math Inequal Appl 20, 583-589.

8. Huang S, Wang Q, Zhang Y (2018) Equivalence on some Rotfel'd type theorems. Linear Multilinear Algebra 66, 1626-1632.

9. Kuai L (2018) An extension of the Fiedler-Markham determinant inequality. Linear Multilinear Algebra 66, 547-553.

10. Lee EY (2011) Extension of Rotfel'd theorem. Linear Algebra Appl 435, 735-741.

11. Lin M, Wolkowicz H (2015) Hiroshima's theorem and matrix norm inequalities. Acta Sci Math (Szeged) 81, 45-53.

12. Lin M (2012) Reversed determinantal inequalities for accretive-dissipative matrices. Math Inequal Appl 15, 955-958.

13. Lin $M$ (2013) Fischer type determinantal inequalities for accretive-dissipative matrices. Linear Algebra Appl 438, 2808-2812.

14. Lin M (2015) Extension of a result of Haynsworth and Hartfiel. Arch Math 104, 93-100.

15. Yang J, Lu L, Chen Z (2019) A refinement of Rotfel'd type inequality for partitioned matrices with numerical ranges in a sector. Linear Multilinear Algebra 67, 1719-1726.

16. Zhang P (2015) A further extension of Rotfel'd theorem. Linear Multilinear Algebra 63, 2511-2517.

17. Zhao J, Ni K (2018) Some extensions of Rotfel'd theorem. Linear Multilinear Algebra 66, 410-417.

18. Zhang F (2011) Matrix Theory, Basic Results and Techniques, 2nd edn, Springer, New York.

19. Zhang F (2015) A matrix decomposition and its applications. Linear Multilinear Algebra 63, 2033-2042. 